University of New Hampshire

University of New Hampshire Scholars' Repository

Space Science Center

Institute for the Study of Earth, Oceans, and

Space (EOS)

2000

\title{
Spectra of a recent bright burst measured by CGRO-COMPTEL: GRB 990123
}

C A. Young

University of New Hampshire - Main Campus

A Connors

Eureka Scientific

K Bennett

ESTEC

W Collmar

Max-Planck-Institut für extraterrestriche Physik

W Hermsen

Space Research Organization of the Netherlands (SRON)

See next page for additional authors

Follow this and additional works at: https://scholars.unh.edu/ssc

Part of the Astrophysics and Astronomy Commons

\section{Recommended Citation}

Spectra of a recent bright burst measured by CGRO-COMPTEL: GRB 990123 Young, C. A. and Connors, A. and Bennett, K. and Collmar, W. and Hermsen, W. and Kippen, R. M. and Kolaczyk, E. D. and Kuiper, L. and McConnell, M. and Miller, R. and Ryan, J. M. and Schönfelder, V. and Williams, O. R. and Winkler, C., AIP Conference Proceedings, 526, 170-174 (2000), DOI:http://dx.doi.org/10.1063/1.1361528

This Conference Proceeding is brought to you for free and open access by the Institute for the Study of Earth, Oceans, and Space (EOS) at University of New Hampshire Scholars' Repository. It has been accepted for inclusion in Space Science Center by an authorized administrator of University of New Hampshire Scholars' Repository. For more information, please contact Scholarly.Communication@unh.edu. 


\section{Authors}

C A. Young, A Connors, K Bennett, W Collmar, W Hermsen, R M. Kippen, E Kolaczyk, L Kuiper, Mark L. McConnell, R S. Miller, James M. Ryan, V Schonfelder, O R. Williams, and C Winkler 


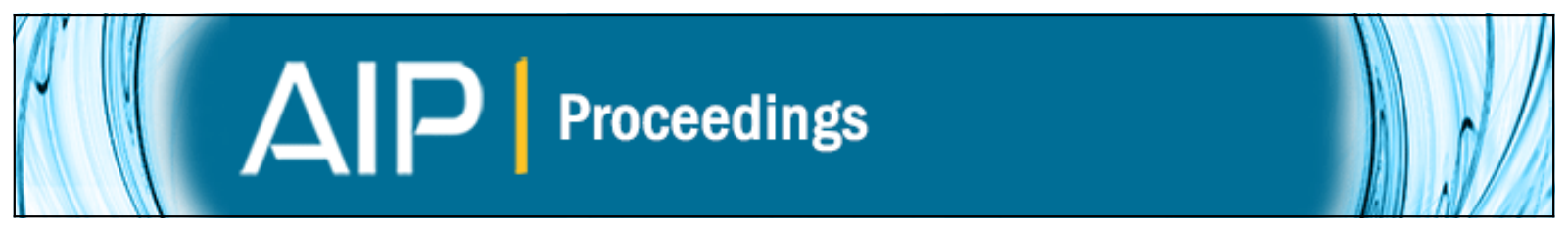

Spectra of a recent bright burst measured by CGRO-COMPTEL: GRB 990123

C. A. Young, A. Connors, K. Bennett, W. Collmar, W. Hermsen, R. M. Kippen, E. D. Kolaczyk, L. Kuiper, M. McConnell, R. Miller, J. M. Ryan, V. Schönfelder, O. R. Williams, and C. Winkler

Citation: AIP Conference Proceedings 526, 170 (2000); doi: 10.1063/1.1361528

View online: http://dx.doi.org/10.1063/1.1361528

View Table of Contents: http://scitation.aip.org/content/aip/proceeding/aipcp/526?ver=pdfcov

Published by the AIP Publishing

\section{Articles you may be interested in}

Power density spectra of gamma-ray bursts

AIP Conf. Proc. 526, 205 (2000); 10.1063/1.1361535

X-ray excesses in GRB spectra

AIP Conf. Proc. 526, 175 (2000); 10.1063/1.1361529

Study of the characteristics of GRB energy spectra

AIP Conf. Proc. 526, 160 (2000); 10.1063/1.1361526

Gamma-ray burst spectroscopy

AIP Conf. Proc. 526, 115 (2000); 10.1063/1.1361518

Testing the intrinsic randomness in the angular distributions of gamma-ray bursts

AIP Conf. Proc. 526, 102 (2000); 10.1063/1.1361516 


\title{
Spectra of a Recent Bright Burst Measured by CGRO-COMPTEL:GRB 990123
}

\author{
C. A. Young ${ }^{1}$, A. Connors ${ }^{2}$, K. Bennett ${ }^{3}$, W. Collmar ${ }^{4}$, \\ W. Hermsen ${ }^{5}$, R. M. Kippen ${ }^{6}$, E. D. Kolaczyk ${ }^{7}$, L. Kuiper ${ }^{5}$, \\ M. McConnell ${ }^{1}$, R. Miller ${ }^{1}$, J. M. Ryan ${ }^{1}$, V. Schönfelder ${ }^{4}$, \\ O. R. Williams ${ }^{3}$, and C. Winkler ${ }^{3}$ \\ ${ }^{1}$ Space Science Center, University of New Hamphsire, Durham NH, USA \\ ${ }^{2}$ Eureka Scientific \\ ${ }^{3}$ Astrophysics, Division, ESTEC, NL-2200 AG Noordwijk, NL \\ ${ }^{4}$ Max-Planck-Institut fur Extraterrestrische Physik, D-85740 Garching, FRG \\ ${ }^{5}$ SRON-Utrecht, Sorbonnelaan 2, NL-3584 Utrecht, $N L$ \\ ${ }^{6}$ CSPAAR, University of Alabama in Huntsville, Huntsville AL, USA \\ ${ }^{7}$ Department of Mathematics and Statistics, Boston University, Boston MA, USA
}

\begin{abstract}
CGRO-COMPTEL measures gamma-ray burst positions, time-histories and spectra in the $0.1-30 \mathrm{MeV}$ energy range, in both imaging "telescope" and single detector "burst spectroscopy" mode. GRB 990123, one of the most recent bright bursts seen by COMPTEL, was caught in the optical while the gamma-ray emission was ongoing. The burst spectral shape can be characterized by a peak in $\nu-F_{\nu}$ just below $1 \mathrm{MeV}$ and a power-law tail above (photon index $\sim-2.4$ ), and flattening below. There is also spectral evolution by downward movement of the peak and/or softening of the power laws. We present light-curves, time resolved spectra and an image map for this burst.
\end{abstract}

\section{INTRODUCTION}

GRB 990123 is the only gamma-ray burst to be simultaneously observed in optical wavelengths. The Burst and Transient Source Experiment (BATSE) on CGRO triggered the Compton Telescope (COMPTEL) on CGRO at $35216.121 \mathrm{~s}$ UT on 23 January 1999 (BATSE trigger \#7343). The burst was located at a zenith angle (w.r.t. CGRO and COMPTEL) pointing of $58.4^{\circ}$. The gamma-ray emission lasted for about $100 \mathrm{~s}$ with the $>1 \mathrm{McV}$ cmission seen by COMPTEL most significant between $18 \mathrm{~s}$ and $46 \mathrm{~s}$ after the BATSE trigger. The Robotic Optical Transient Search Experiment (ROTSE) detected optical emission during and after the gamma-ray emission. ROTSE made 6 measurements starting $22.2 \mathrm{~s}$ after the BATSE trigger.

CP526, Gamma-Ray Bursts: $5^{\text {th }}$ Huntsville Symposium, edited by R. M. Kippen, et al. (C) 2000 American Institute of Physics 1-56396-947-5/00/\$17.00 


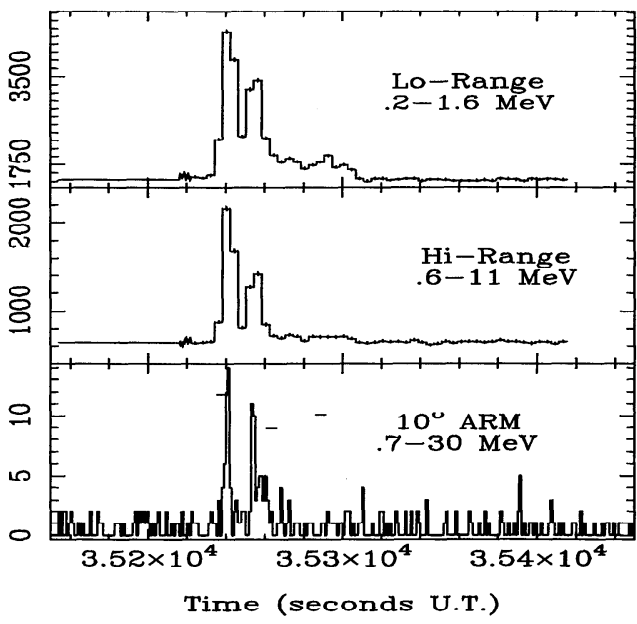

COMPTEL detected the second and third peaks of GRB 990123 in both its imaging telescope ("double scatter"; $0.75-30 \mathrm{MeV}$ ) and non-imaging burst-spectroscopy ("burst mode"; 0.3-1.5 MeV and 0.6-10 MeV; see Schönfelder et al. 1993) modes. Telescope position constraints were broadcast (via GCN/BACODINE) about 10 minutes after burst onset and preliminary light-curves were posted soon thereafter (Young et al. 1999; Connors et al. 1999).

In the imaging telescope mode, COMPTEL provides detailed information on individual time-tagged photons with $\frac{1}{8} \mathrm{~ms}$ time resolution. Because of the small effective area and limited telemetry of the telescope mode, however, only $\sim 200$ burst events were recorded. In spectroscopy mode the effective area was roughly two orders of magnitude greater, and the dead time was negligible. The COMPTEL light-curves are displayed in Fig. 1.

\section{TELESCOPE MODE}

The spectra from the two modes were handled differently. For the $0.75-30 \mathrm{MeV}$ telescope data, one selects only events whose event circle falls within a certain angle of the source position ("angular resolution measure", or ARM; Schönfelder et al. 1993), both reducing background and providing a nearly diagonal response (e.g., Kippen et al. 1998). For the telescope spectrum displayed in Fig. 2, a $10^{\circ}$ ARM limit was used. The $32.768 \mathrm{~s}$ integration interval of $(35229.452 \mathrm{~s}, 35262.22 \mathrm{~s})$ was chosen both to cover all the significant gamma-ray emission and to allow an accurate live time calculation. The background data were taken 15 orbits prior to the burst (see Kippen et al. 1998). The data were fit to a simple power-law via a forward folding technique. The best-fit is $2.0 \perp 0.4(E / 1 \mathrm{MeV})^{-3.33 \pm 0.4}$ photons $/ \mathrm{cm}^{2}$-s-MeV, giving a total fluence of $(0.98 \pm 0.5) \times 10^{-4} \mathrm{ergs} / \mathrm{cm}^{2}(0.75-30 \mathrm{MeV})$. However, a turnover at or below $1 \mathrm{MeV}$ is also consistent with the telescope data. 


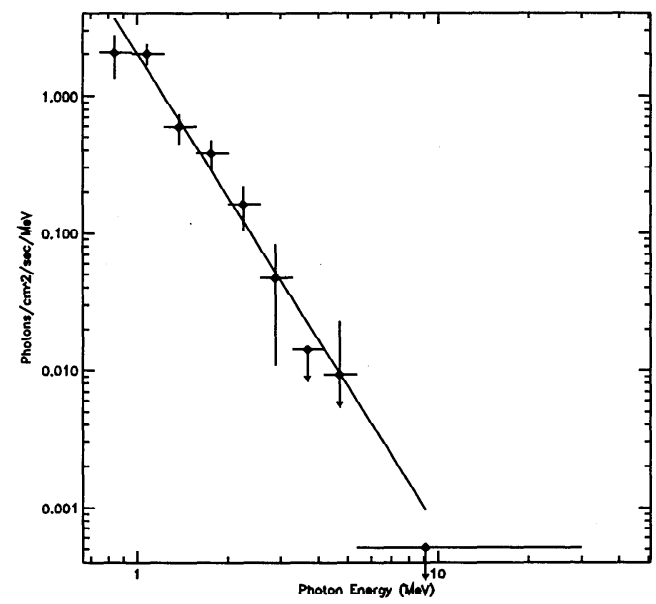

FIGURE 2. The best-fit power-law spectrum is $2.0 \pm 0.4(E / 1 \mathrm{MeV})^{-3.33 \pm 0.4}$ photons $/ \mathrm{cm}^{2}-\mathrm{s}-\mathrm{MeV}$, giving a total $\mathrm{flu}-$ ence of $(0.98 \pm 0.5) \times 10^{-4} \mathrm{ergs} / \mathrm{cm}^{2}$ (0.75-30 MeV). IIowever, a turnover at or below $1 \mathrm{MeV}$ is also consistent with these telescope data.

\section{BURST MODE}

The single detector count spectra obtained in "spectroscopy mode" were processed as follows: the background was estimated from a spectrum of $140 \mathrm{~s}$ duration starting $202 \mathrm{~s}$ prior to the BATSE trigger (at $35216 \mathrm{~s}$ ). Eight high range detector $(0.6-10.0 \mathrm{MeV})$ spectra (4 s integration time each) covering a $32 \mathrm{~s}$ time interval $(35230.2 \mathrm{~s}, 35262.3 \mathrm{~s})$, were background subtracted and summed. The spectral fitting of these time-averaged data was performed by convolving a trial photon spectrum with the detcctor response matrix to produce a count spectrum. We used a $\chi^{2}$ statistic to compare the model spectrum with the data. We first assumed a single power law for the spectrum between 0.9 and $5.0 \mathrm{MeV}$. As shown in Fig. 1 there is no significant signal above $4 \mathrm{MeV}$. The best fit parameters for the single power law in this energy range are: normalization $=(1.37 \pm 0.10)$ photons $/\left[\mathrm{cm}^{2}-\mathrm{sec}-\mathrm{MeV}\right]$ at $1 \mathrm{MeV}$; and index $=(-2.63 \pm 0.16)$. The fluence $(0.9-5 \mathrm{MeV}, 32 \mathrm{sec})$ is $7.86 \times 10^{-5}$ $\mathrm{erg} / \mathrm{cm}^{2}$. We note a clear break (Briggs et al. 1999) in the spectrum below $0.9 \mathrm{MeV}$, where the spectrum becomes flatter. Also, preliminary analysis of the low range $(0.3-1.5 \mathrm{MeV})$ spectroscopy data covering the same $32 \mathrm{~s}$ time interval indicates a single power law with index -2.0 .

Due to evidence for spectral evolution (Briggs et al. 1999) with a break in COMPTEL's energy range, one goal of this analysis was to produce time resolved spectra of this burst. Traditional spectral analysis techniques (like those applied above) use a parametric approach assuming a particular model and approximately account for the Poisson nature of the data using $\chi^{2}$ type approximations or data transformations. These techniques thus require high statistics and so don't allow for fine time binning of our data. We have begun the implementation of a newly developed technique that addresses some of these shortcomings enabling the use of finer time resolution. Nowak and Kolaczyk (1999) have presented a method for deconvolving spectra that is both non-parametric and explicitly handles Poisson data. We 

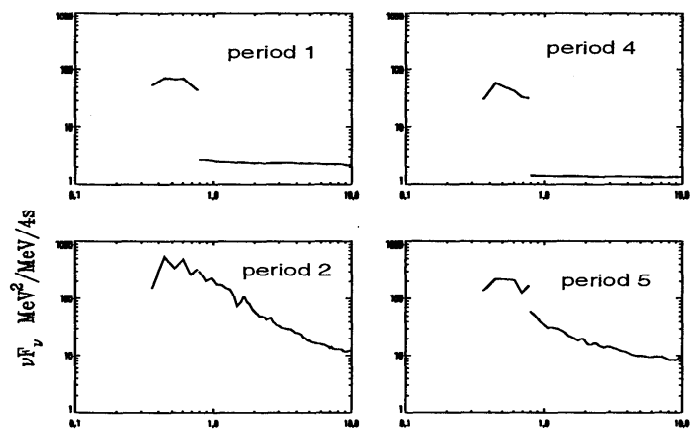

FIGURE 3. Time resolved $\nu-F_{\nu}$ spectra of 6 consecutive $4 \mathrm{~s}$ intervals of GRB 990123, showing qualitative evidence for spectral evolution. The deconvolution was performed using a

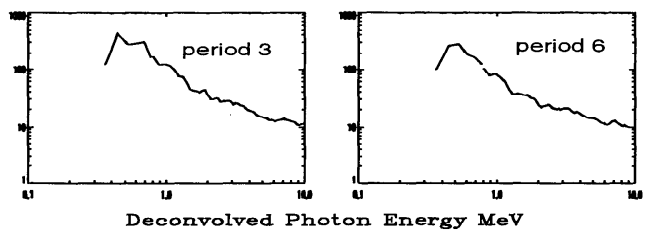
non-parametric approach.

divided the low and high range burst data into seven $4 \mathrm{~s}$ time intervals. Using this non-parametric technique we deconvolved these spectra with the instrumen$\mathrm{t}$ response. Though only preliminary, Fig. 3 shows qualitative spectral structure consistent with the previous analysis with a spectral break below $1 \mathrm{MeV}$. The time periods displayed don't show any spectral evolution but they are consistent with the comparable times shown in Briggs et al. (1999).

\section{IMAGE MAP}

GRB 990123 was so bright in $\mathrm{MeV}$ gamma-rays that COMPTEL was able to image it in a 10 sigma detection. (The preliminary COMPTEL detection, broadcast about 11 minutes after the BATSE trigger, was 8.2 sigma.) Fig. 4 shows the combined COMPTEL and BATSE 1, 2, and 3 sigma location contours. The IPN, BeppoSAX X-ray, prompt Optical (ROTSE), fading optical, and radio counterpart locations are included on the map.

\section{CONCLUSIONS}

For GRB 990123, observed in the optical during the gamma-ray emission, one notes that the optical flux peaks after the brightest gamma-ray portion and is not a simple extrapolation of the $\mathrm{MeV}$ flux. Some have noted this may be a signature of self-absorption; while others suggest this shows the optical to have come from a separate component (Briggs et al. 1999). Traditional analysis techniques show consistent results for the burst and telescope data from COMPTEL. A preliminary non-parametric analysis is consistent with other methods and shows qualitative 


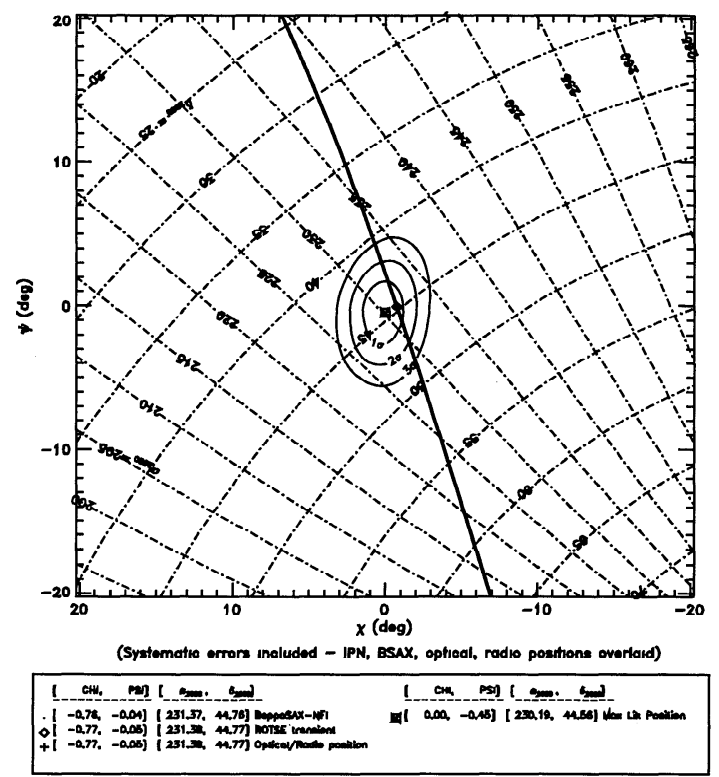

FIGURE 4. The combined COMPTEL and BATSE Huntsville 1,2 and 3 sigma location contours (systematic unccrtainties included), along with the IPN timing arc (Hurley, Feroci et al GCN 222), and indications of the BeppoSAX X-ray, prompt optical (ROTSE), fading optical, and radio counterparts.

evidence for a spectral break just under $1 \mathrm{MeV}$. Further investigation of this new technique will allow for quantitative results.

\section{ACKNOWLEDGMENTS}

The COMPTEL project is supported in part through NASA grant NAS 5-26646, DARA grant 50 QV 90968, and the Netherlands Organization for Scientific Research (NWO). AC is supported in part through the hospitality of Wellesley College and NASA grant NAG5-7984. MM is supported in part through NASA grant NAG5-7829.

\section{REFERENCES}

1. Akerlof, C. W., et al., GCN 205 (1999).

2. Briggs, M. S., ct al., $A p J$ 524, 82 (1999).

3. Connors, A., et al., GCN 230, 237 (1999).

4. Hurley, K., et al., GCN 222 (1999).

5. Kippen, R. M., et al., Adv. Space. Res. 22 (7), 1097-1100 (1998).

6. Loredo, T. J., in Statistical Challenges in Modern Astronomy, eds. G. J. Babu \& E. D. Fiegelson, Springer-Verlag, New York, 1999.

7. Nowak, R. D., Kolaczyk, E. D., IEEE Trans. Inform. Theory, Sept. (1999).

8. Schönfelder, V., et al., ApJS 86, 629 (1993). 